\title{
Complete intraperitoneal displacement of a double J stent: A first case
}

\author{
Filippo Maria Turri ${ }^{1}$, Francesca Manassero ${ }^{1}$, Andrea Mogorovich ${ }^{1}$, Maurizio De Maria ${ }^{1}$, \\ Andrea Falleni ${ }^{2}$, Cesare Selli ${ }^{1}$ \\ ${ }^{1}$ Department of Translational Research, Section of Urology, University of Pisa, Italy; \\ ${ }^{2}$ Department of Emergency Medicine, Section of Diagnostic Radiology, AOUP, Pisa, Italy.
}

\begin{abstract}
Summary Objectives: Ureteral double-J stents are known to migrate proximally and distally within the urinary tract, while perforation and stent displacement are uncommon. Possible mechanisms of displacement are either original malpositioning with ureteral perforation or subsequent fistula and erosion of the excretory system, due to infection or long permanence of the device. We present the unique case of complete intraperitoneal stent migration in a 59-year-old caucasian male without evidence of urinary fistula at the moment of diagnosis, so far an unreported complication.

Materials and Methods: Eight months after the placement of a double-J stent for lower right ureteral stricture at a district hospital, the patient came at our observation for urosepsis and hydro-uretero-nephrosis. A CT scan demonstrated intraperitoneal migration of the stent outside the urinary tract. Cystoscopy failed to visualize the lower extremity of the stent, a percutaneous nephrostomy was placed to drain the urinary system and the stent was removed through a small abdominal incision on the right lower quadrant.

Results: In our case we presume that during the positioning manoeuvre the guide wire perforated simultaneously the lower ureteral wall and the pelvic peritoneum, and that once the upper end of the stent was coiled, the lower extremity was also attracted intraperitoneally. The lack of pain due to the spinal lesion concurred to this unusual complication.

Conclusions: We must be aware that ureteral double J stents may be found displaced even inside the peritoneal cavity, and that the use of retrograde pyelography during placement is of paramount importance to exclude misplacement of an apparently normally coiled upper extremity of the stent.
\end{abstract}

KEY WORDS: Ureteral stent; Stent displacement; Endourology; Abdominal imaging; CT scan.

Submitted 15 October 2014; Accepted 31 December 2014

\section{INTRODUCTION}

First described in 1967 by Zimskind et al. (1), ureteral double J (DJ) stents are widely employed in urology. The most frequent complications are flank pain, irritative voiding symptoms, urinary tract infections, encrustation, stent displacement, hematuria, obstruction, malpositioning, iatrogenic lesions and forgetfulness of the stent (2). More peculiar complications are knotting (3) or rupture (4) of the stent, ureteral wall erosion and stent migration outside of the urinary tract (5-7).

\section{Materials and Method}

A 59-year-old man with motor and sensitive deficiency secondary to cervical spine trauma and with a history of previous transurethral resection (TUR) for non muscleinvasive bladder cancer, underwent placement of a DJ stent for a lower right ureteral stricture at a district hospital in April 2013. Eight months later he came to our observation for urosepsis. Physical examination was negative. Sonography revealed right hydro-uretero-nephrosis with no evidence of lithiasis. Abdominal CT scan demonstrated the right ureteral stent displaced outside the urinary tract, intraperitoneally for its whole length (Figures 1-3). At cystoscopic examination it was impossible to visualize the stent. A right percutaneous nephrostomy was placed, purulent urine was drained and an antegrade contrast study showed a dilated ureter with blind end at the level of L 5, without extravasation of the contrast medium.

\section{Figure 1.}

Basal CT scan: right hydronephrosis and upper coil of the stent located inside the peritoneal cavity.

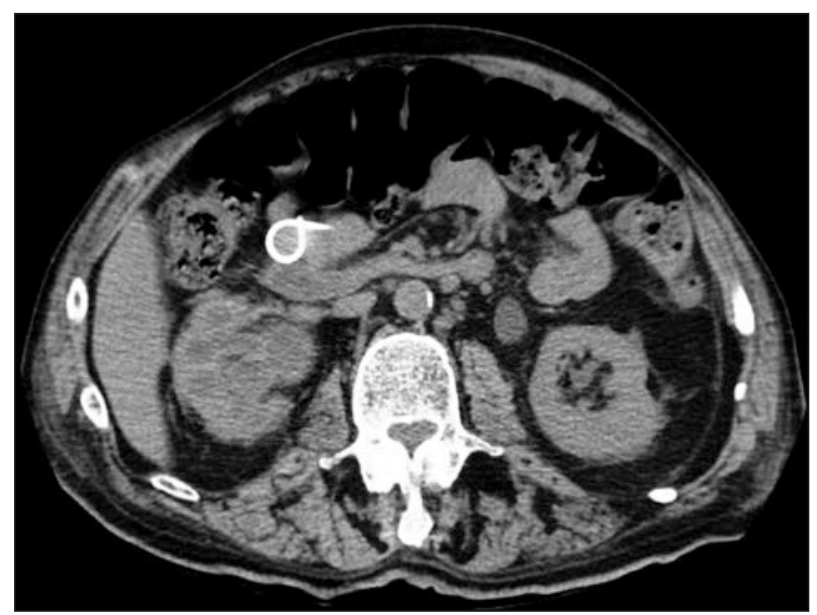


Figure 2.

$3 D$ volume rendering of $C T$ scan revealing the upper coil of the stent displaced anteriorly to the kidney and the lower coil in the right lower quadrant.

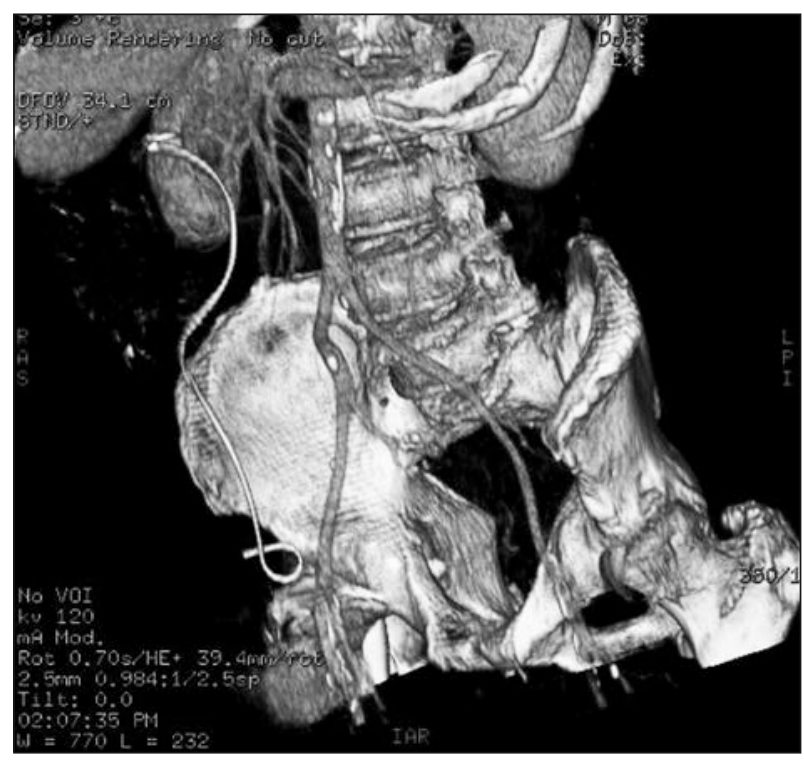

\section{Figure 3.}

Oblique reconstruction of the uro-CT scan revealing a tortuous and dilated right ureter not containing the ureteral stent.

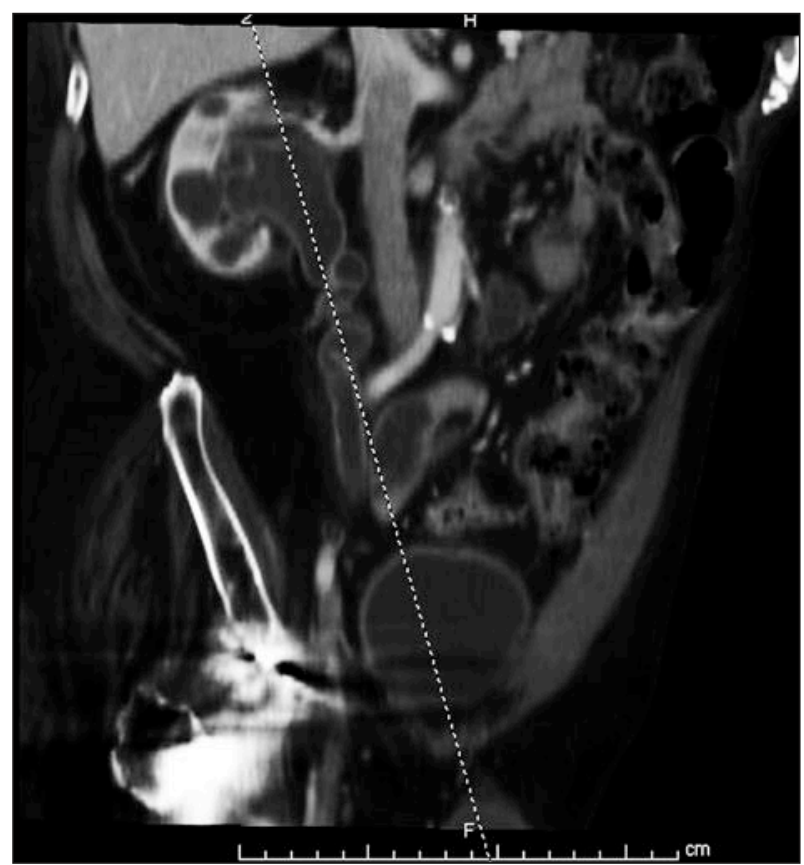

\section{RESULTS}

Following urine drainage the patient became afebrile and four days later surgical exploration was performed: the intraperitoneal stent was extracted through a small muscle splitting incision in the right lower quadrant (Figure 4)

One month after nuclear renography showed a glomerular filtration rate (GFR) of $45 \mathrm{ml} / \mathrm{min}$ for the left kidney and of $8 \mathrm{ml} / \mathrm{min}$ for the right kidney.
Figure 4.

Retrieval of the intraperitoneal stent through a short muscle-splitting incision in the right lower quadrant.

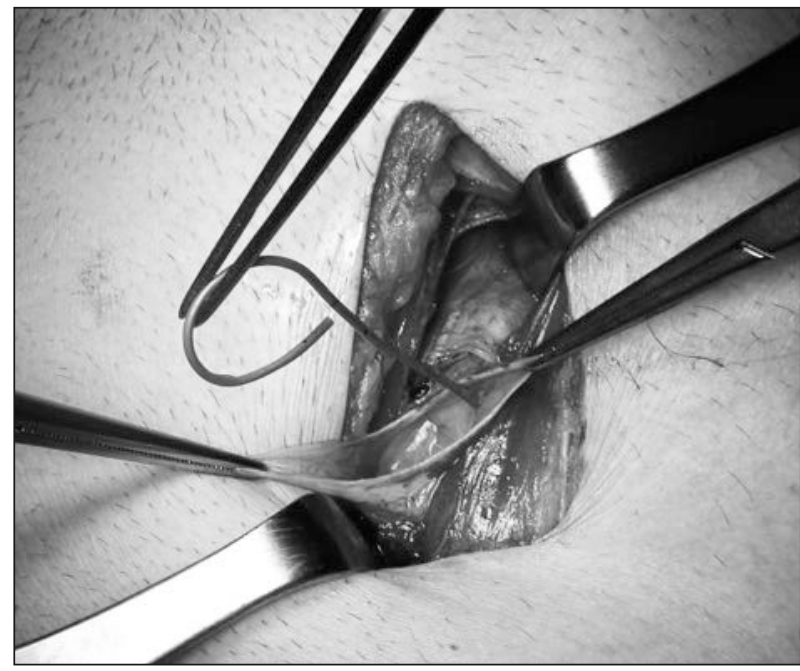

Given the impaired general conditions of the patient, who had developed a subsequent cerebral hemorrhage, and the severely reduced GFR of the right kidney, it was decided to leave an indwelling nephrostomy to avoid obstruction, without considering ureteral reconstruction.

\section{Discussion}

While cranial or caudal migration of ureteral DJ stents is a relatively common event, their displacement outside the urinary tract occurs infrequently, and has been described as retroperitoneal and incomplete (5-7); migration has been reported even inside the lumen of the vena cava (8). The possible mechanisms of stent displacement are either original malpositioning following ureteral perforation or subsequent erosion of the excretory system, due to infection or long permanence of the device.

Although in one case report the upper tip of a DJ was displaced inside the peritoneal cavity through a fistula of the upper pole of the kidney with concomitant urinary peritonitis (9), to the best of our knowledge this is the first report of complete intraperitoneal stent migration without evidence of urinary extravasation or fistula at the time of diagnosis.

Since the intraperitoneal stent appeared clean and patent, we presume that during the positioning manoeuvre the guide wire perforated simultaneously the lower ureteral wall and the pelvic peritoneum, and that once the upper end of the stent was coiled, the lower extremity was also attracted intraperitoneally. The lack of pain due to the spinal lesion probably concurred to this unusual complication and such a late presentation. A small open surgical access on the right lower quadrant was preferred to a laparoscopic approach because we felt that it was faster and less invasive overall.

\section{Conclusions}

Clinicians must be aware that ureteral DJ stents may be found displaced even inside the peritoneal cavity, and that 
the use of retrograde pyelography during stent placement is of paramount importance to exclude misplacement.

\section{REFERENCES}

1. Zimskind PD, Fetter TR, Wilkerson JL. Clinical use of long-term indwelling silicone rubber ureteral splints inserted cystoscopically. J Urol. 1967; 97:840-4.

2. Christopher Wolter, Roger Dmochowski. Insertion of Double-J Stent. In: Hashim H, Abrams P, Dmochowski R (Ed), The Handbook of Office Urological Procedures. London: Springer-Verlag, 2008; 142.

3. Picozzi S, Carmignani L. A knotted ureteral stent: a case report and review of the literature. Urol Ann. 2010; 2:80-2.

4. Witjes JA. Breakage of a silicone double pigtail ureteral stent as a long-term complication. J Urol. 1993; 150:1898-9.
5. Abraham G, Das K, George D. Retroperitoneal migration of a double-J stent: an unusual occurrence. J Endourol. 2011; 25:297-9.

6. Shivde SR, Joshi P, Jamkhandikar R. Extrusion of a double J stent: a rare complication. Urology 2008; 71:814-5.

7. Ioannis C, Ioannis Z, Evangelos I, et al. Retrovesical migration of malpositioned double-J ureteral stent. Int Urol Nephrol. 2003; 35:325-6.

8. Falahatkar S, Memmati H, Gholamjani MK. Intracaval migration: an uncommon complication of ureteral double-J stent placement. J Endourol. 2012; 26:119-21.

9. Ivica S, Dragan S. Long-term indwelling double-J stents: bulky kidney and urinary bladder calculosis, spontaneous intraperitoneal perforation of the kidney and peritonitis as a result of "forgotten" double-J stent. Vojnosanit Pregl. 2009; 66:242-4.

\section{Correspondence}

Filippo Maria Turri, MD

fm.turri@gmail.com

Francesca Manassero, MD

francy_manassero@hotmail.com

Andrea Mogorovich, MD

mogorovich@hotmail.it

Maurizio De Maria, MD

m.demaria@ao-pisa.toscana.it

Cesare Selli, MD, Professor of Urology (Corresponding Author)

c.selli@med.unipi.it

Department of Translational Research, Section of Urology

University of Pisa, via Paradisa I-56124 Pisa, Italy

Andrea Falleni, MD

a.falleni@ao-pisa.toscana.it

Department of Emergency Medicine, Section of Diagnostic Radiology

AOUP, Pisa, Italy 